\title{
GENERATION OF STRONG TOROIDAL MAGNETIC FIELD NEAR THE BOTTOM OF THE SOLAR CONVECTIVE ZONE
}

\author{
V.N.KRIVODUBSKIJ \\ Kiev University Astronomical Observatory \\ Observatorna st. 3, Kiev-53 254053, Ukraine
}

\begin{abstract}
The generation mechanism of the toroidal magnetic field by the angular velocity radial gradient acting on the relict poloidal magnetic field on the boundary between the convective and radiative zones is proposed. The magnetic induction magnitude of the toroidal field reaches about $2 \times 10^{6} \mathrm{G}$, the limiting effect of the magnetic buoyancy being taking into accaunt. This value conforms to the estimation of toroidal field obtained from helioseismological data.
\end{abstract}

Studying the helioseismological data, recently Dziembowski and Goode (1989) have shown that there is strong axisymmetric quadrupole toroidal field of magnetic induction $(2 \pm 1) \times 10^{6} \mathrm{G}$ centred near the base of the convective zone. The question about the origin of this magnetic field arises. In connection with fast upwelling to the surface of intensive magnetic field due to the magnetic buoyancy, it is necessary to show also how this field may reserve inside the Sun during long time. Here it is made the attempt to solve this problem with help proposed by us the mechanism of generation of the strong toroidal magnetic field in deep layers of the sun.

According this mechanism, the radial gradient of angular velocity acts on the relict poloidal magnetic field ${ }_{B}$ and excites the toroidal magnetic field $\underline{B}_{t}=B_{\varphi} \underline{i} \varphi p$ the magnitude of which is limited by ohmic dissipation and magnetic buoyancy (Dudorov et al. 1989; Krivodubskij 1990)

$\partial \underline{B}_{t} / \partial t=r \sin \theta\left(\underline{B}_{p} \underline{\underline{i}}_{r} \nabla \Omega\right) \underline{i}+\nu_{m} \Delta \underline{B}_{t}+\operatorname{rot}\left(\underline{V}_{b} x \underline{B}_{t}\right) \cdot(1)$ 
Here $\nabla \Omega=\Delta \Omega / \Delta x$ is the radial gradient of angular velocity, $\Delta \Omega$ is the increment in the angular velocity over step $\Delta r$ along solar radius $R_{\odot}, \nu$ is the magnetic viscosity, $\underline{V}_{b} \simeq U_{T}(\lambda / a)^{2}\left(B^{2} \varphi / 8 \pi P_{e}\right)_{I_{r}}^{m}$ is the velocity of upwelling of a toroidal magnetic flux tube (MFT) in the region of the radiative energe transfer, $\underline{i}_{\varphi}$ and $\dot{i}_{r}$ are the ozimuthal and radial unit vectors in the ${ }^{-r}$ spherical coordinat system, $\theta$ is the complement of the latitude, $r$ is the distance from the centre of the Sun, $\lambda$ is the temperature scale height, a is the transverse radius of the $\mathrm{MTT}$ $U_{T}$ is the mean velocity of upward transport of thermal energy, $P$ is the electron pressure.

on the present stage of solar evolution the magnetic buoyancy plays the main role in the constraining of the magnetic induction of the toroidal field. Then from the condition of stationarity, $\partial \mathrm{B} \varphi / \partial t=0$, neglecting ohmic dissipation, from Eq. (1) we obtain the following expression for maximum value of the resulting stationary toroidal field (Krivodubskij 1990, 1992):

$\max \left|B_{\varphi}\right|=B_{\varphi}^{0} \simeq\left(\frac{\Gamma}{\gamma}\right)^{1 / 3}=\left[r \frac{\Delta \Omega}{\Delta r^{-}} B_{p} \frac{8 \pi P_{e} L}{U_{T}}\left(-\frac{a}{\lambda}-\right)^{2}\right]^{1 / 3}(2)$

which is excited in the radiative zone at the distance $r$ from the centre of the Sun. Here $r=r B \Delta \Omega / \Delta r$ is the generation parameter, $\gamma=U_{\pi} / 8 \pi P I^{p}(\lambda / a)^{2}$ is the parameter of the rate of loss of induction due to magnetic buoyancy, I is the space scale of the magnetic field. The characteristic time of reaching of stationary magnetic induction $\mathrm{B}_{\varphi}^{\circ}$ is $t^{0}=B_{\varphi}^{0} / \Gamma=\left(1 / \gamma \Gamma^{2}\right)^{1 / 3}$.

Dziembowski, Goode and Libbrecht (1989) from the oscillation data of Libbrecht (1989) found that there is the transition from differential rotation to riget rotation at the convective zone boundary with the radiative interior. Our analysis of the dependence $\Omega(r, \theta)$ showed that the maximum jump of angular velocity, $\Delta \Omega \cong 1 \times 10^{-7} \mathrm{rad} / \mathrm{s}$, occurs in the equatorial plane along solar radius on short piece length of radius, $\Delta r \simeq 0,74 R_{\odot}-0,67 R_{0}=$ $0,07 R_{\odot} \simeq 5 \times 10^{9} \mathrm{~cm}$. Id est, there is the sharp radial gradient of angular velocity, $\Delta \Omega / \Delta \mathrm{r} \cong 2 \mathrm{x}_{1} 0^{-17} \mathrm{rad} / \mathrm{s} \mathrm{cm}$, in the sufficient narrow layer $\Delta r$. This evidences that at transition from convective zone to radiative interior there is the favourable conditions for 
effective generation of toroidal magnetic field in accordance with the mechanism above-mentioned.

When for physical parameters $U_{m}, \lambda, P_{e}$ at the upper part of radiative zone we take values from internal structure model of the Sun (Allen 1973); for space parameters of toroidal field $\mathrm{B}_{\varphi} \varphi$ we take $I=2 a=\Delta r$; and for quadrupole mode of the observed poloidal component $B_{p}$ we take value $\sim 1 G$ in accordance with Stenflo (1988), then we obtain the following estimates for the parameter generation, $r$, and the parameter of loss of magnetic induction, $\gamma$, near the radiative zone top $\left(r \simeq 0,7 R_{\odot}\right)$ at narrow layer $\Delta r \cong 5 \times 10^{9} \mathrm{~cm}: \quad \Gamma \simeq 1 \times 10^{-6} \mathrm{G} / \mathrm{s}, \gamma \approx$ $2 \times 10^{-25} \mathrm{G}^{-2} \mathrm{~s}^{-1}$. For relatevely short time space to $\simeq 70,000$ years magnetic induction of excited toroidal field reaches maximum stationary value $\mathrm{BO}_{\psi} \simeq 2 \times 10^{6} \mathrm{G}$ (at the diameter of $\mathrm{MFT} \mathrm{d}_{t} \leqslant 5 \times 10^{9} \mathrm{~cm}$ which are oriented along the parallels of solar latitude with opposite sign on either side of equatorial plane). This value agrees with the helioseismological finding of Dziembowski and Goode (1989). The presence of such intensive toroidal field near convective zone bottom is essential for maintenance of dynamo-process in the region of turbulent convection.

\section{REFERENCES}

Allen, C.W. 1973, Astrophysical Quantities, Athlone Press, London.

Dudorov, A.E., Krivodubskij, V.N., Ruzmaikina, T.V. and Ruzmaikin, A.A. 1989, Astron. Zh., 66, 809 (Sov. Astron., 33, 420).

Duvall Jr., T.I. et al. 1984, Nature, 310, 22.

Dziembowski, W.A., Goode, P.R. 1989, Ap.J., 347, 540. Dziembowski, W.A., Goode, P.R. 1990, Inside the Sun, Dordrecht, p.341.

Dziembowski, W.A., Goode, P.R., Libbrecht, K.G. 1989, Ap. J. (Ietters), 337, I53.

Krivodubskij, V.N. 1990, Vestn. Kiev. Univ. Astronomia, 32, 3 (Russian).

Krivodubskij, V.N. 1992, Visn. Kyijv. Univ. Fiz.-mat. nauky, 3, 84 (Ukrainian).

Iibbrecht, K.G. 1989, Ap. J. 336, 1092.

Stenflo, J.0. 1988. Ap. Space Sci., 144, 321. 